\title{
The hot gas content of fossil galaxy clusters
}

\author{
G. W. Pratt ${ }^{1}$, E. Pointecouteau ${ }^{2,3}$, M. Arnaud ${ }^{1}$, and R. F. J. van der Burg ${ }^{1}$ \\ ${ }^{1}$ Laboratoire AIM, IRFU/Service d'Astrophysique - CEA/DRF - CNRS - Université Paris Diderot, Bât. 709, CEA-Saclay, \\ 91191 Gif-sur-Yvette Cedex, France \\ e-mail: gabriel.pratt@cea.fr \\ 2 CNRS, IRAP, 9 Av. colonel Roche, BP 44346, 31028 Toulouse Cedex 4, France \\ 3 Université de Toulouse, UPS-OMP, IRAP, 31028 Toulouse Cedex 4, France
}

Received 9 March 2016 / Accepted 23 April 2016

\begin{abstract}
We investigate the properties of the hot gas in four fossil galaxy systems detected at high significance in the Planck Sunyaev-Zeldovich (SZ) survey. XMM-Newton observations reveal overall temperatures of $k T \sim 5-6 \mathrm{keV}$ and yield hydrostatic masses $M_{500, \mathrm{HE}} \gtrsim$ $3.5 \times 10^{14} M_{\odot}$, confirming their nature as bona fide massive clusters. We measure the thermodynamic properties of the hot gas in X-rays (out to beyond $R_{500}$ in three cases) and derive their individual pressure profiles out to $R \sim 2.5 R_{500}$ with the $\mathrm{SZ}$ data. We combine the X-ray and SZ data to measure hydrostatic mass profiles and to examine the hot gas content and its radial distribution. The average Navarro-Frenk-White (NFW) concentration parameter, $\left\langle c_{500}\right\rangle=3.2 \pm 0.4$, is the same as that of relaxed "normal" clusters. The gas mass fraction profiles exhibit striking variation in the inner regions, but converge to approximately the cosmic baryon fraction (corrected for depletion) at $R_{500}$. Beyond $R_{500}$ the gas mass fraction profiles again diverge, which we interpret as being due to a difference in gas clumping and/or a breakdown of hydrostatic equilibrium in the external regions. Our observations point to considerable radial variation in the hot gas content and in the gas clumping and/or hydrostatic equilibrium properties in these fossil clusters, at odds with the interpretation of their being old, evolved, and undisturbed. At least some fossil objects appear to be dynamically young.
\end{abstract}

Key words. galaxies: clusters: intracluster medium - X-rays: galaxies: clusters

\section{Introduction}

When Ponman et al. (1994) first looked at the X-ray source RX J1340.6+4018, they found an apparently isolated elliptical galaxy with an extended group-scale X-ray emission. To explain its unusual characteristics, they suggested that it was the result of the coalescence of a group of galaxies into a single giant elliptical, in the process retaining the X-ray emission characteristic of the original halo. Such objects would have formed long ago, leading Ponman et al. to coin the term "fossil galaxy group" to describe them.

Jones et al. (2003) defined a fossil group as having a magnitude gap of $\Delta m_{12} \geq 2$ in the $R$ band between the brightest and the second brightest galaxy within $0.5 R_{200}{ }^{1}$, and an X-ray luminosity of $L_{\mathrm{X} \text {,bol }} \geq 5 \times 10^{41} h_{70}^{-2} \mathrm{erg} \mathrm{s}^{-1}$. The X-ray criterion ensures a group-scale halo, while the optical criterion ensures that there are no $L^{*}$ galaxies inside the radius for orbital decay by dynamical friction. Other authors have modified the optical criterion; for instance, Voevodkin et al. (2010) use a criterion of $\Delta m_{12} \geq 1.7$. We use the Voevodkin et al. definition here.

A key characteristic of the classification scheme advanced by Jones et al. (2003) is that the X-ray criterion is a lower limit. Indeed, a study of the Millennium Simulation has indicated that fossils may be found in significant numbers (3-4\% of the population) even in massive systems (Dariush et al. 2010). Such high-mass systems are of particular interest as they could potentially be the ultimate manifestation of gravitational collapse, while also being sites where non-gravitational effects are

\footnotetext{
${ }^{1} R_{\delta}$ is the radius within which the total density is $\delta$ times the critical
} density at the redshift of the object. minimised. However, whether low- and high-mass fossil systems actually have a common origin is a subject of some debate (e.g., Dariush et al. 2010; Harrison et al. 2012).

There are very few studies of high-mass fossil systems (although Sun et al. 2004 and Khosroshahi et al. 2006 have studied massive groups). The unique properties of the Planck Sunyaev-Zeldovich (SZ) survey make it an ideal resource for finding such objects. The first Planck SZ catalogue (PSZ1, Planck Collaboration XXIX 2014) contains 813 confirmed clusters with known redshifts, of which $779(\sim 96 \%)$ have $M_{500}^{Y_{z}}>2 \times$ $10^{14} M_{\odot}{ }^{2}$. Here we discuss the X-ray and SZ properties of four fossil clusters detected at high significance in the Planck PSZ1 survey. Combining XMM-Newton X-ray and Planck SZ observations, we are able to detect the intra-cluster medium (ICM) out to well beyond $R_{500}$. Despite their very similar X-ray temperatures and total masses, we find a surprising variety in their hot gas content. We adopt a $\Lambda$ CDM cosmology with $H_{0}=70 \mathrm{~km} \mathrm{~s}^{-1} \mathrm{Mpc}^{-1}$, $\Omega_{\mathrm{M}}=0.3$, and $\Omega_{\Lambda}=0.7$; uncertainties are quoted at the $68 \%$ confidence level.

\section{The data}

We first cross-correlated the PSZ1 with three fossil catalogues: Harrison et al. (2012), Voevodkin et al. (2010), and Zarattini et al. (2014). Imposing a mass limit of $M_{500}^{Y_{z}}>3.75 \times$ $10^{14} M_{\odot}$ to select cluster-scale objects, we found three fossil

2 For this initial selection we use the SZ mass $M_{500}^{Y_{z}}$, calculated using the redshift and the SZ mass proxy $Y_{z}$, as described in PSZ1. 

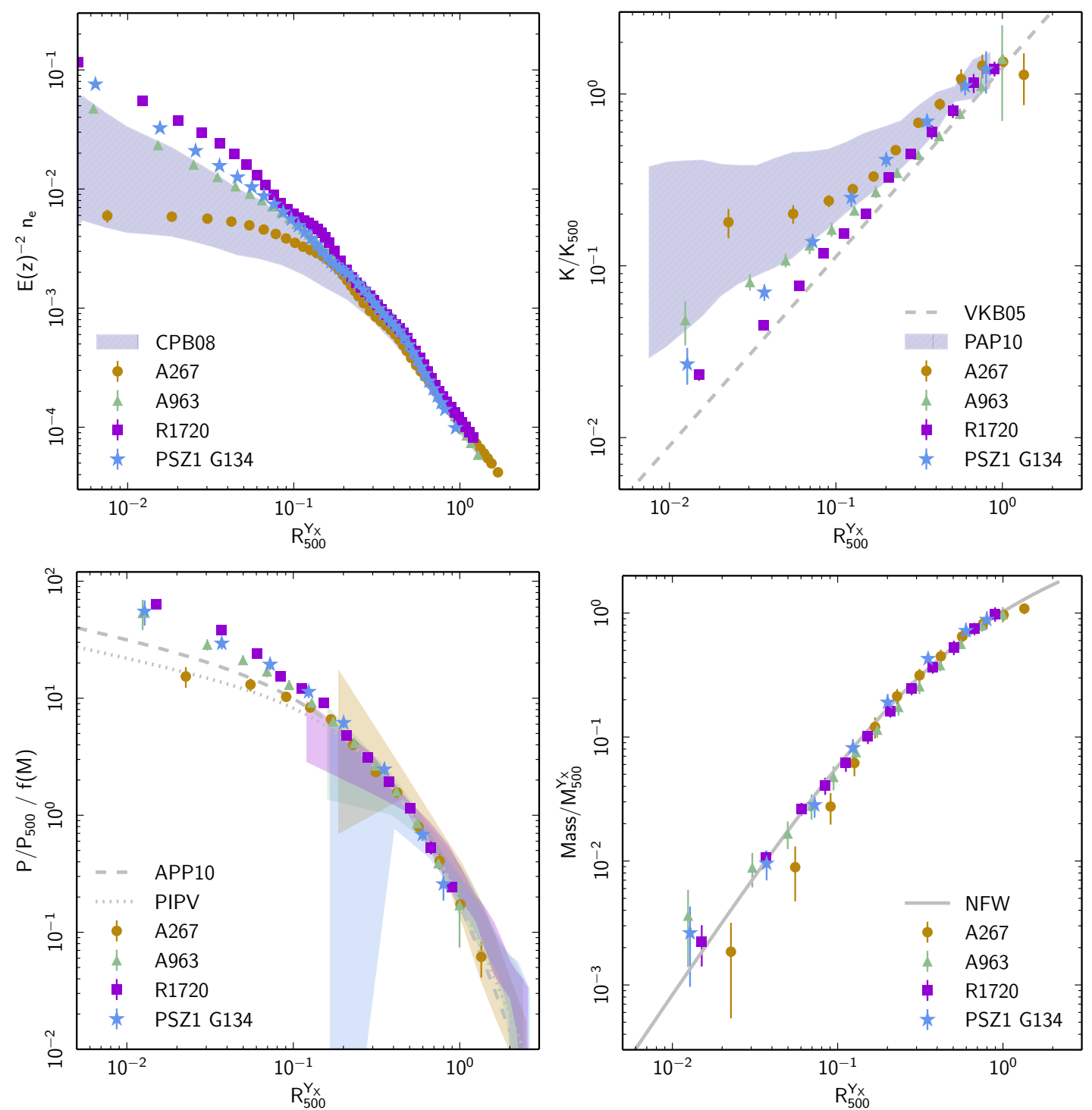

Fig. 1. Top left: scaled density profiles compared to the $1 \sigma$ dispersion of the REXCESS sample (Croston et al. 2008). Top right: scaled entropy profiles compared to the $1 \sigma$ dispersion of the REXCESS sample (Pratt et al. 2010); the dashed line is the baseline gravitational entropy profile of Voit et al. (2005). Bottom left: scaled pressure profiles (points: X-ray, envelopes: SZ) compared to the universal pressure profile of Arnaud et al. (2010) and the mean stacked profile of Planck Collaboration Int. V (2013). $f(M)$ is a (small) correction for the mass dependence in the pressure profile shape (see Arnaud et al. 2010). Bottom right: scaled hydrostatic mass profiles compared to an NFW profile with $c=3.5$, for illustration only. Density, entropy, pressure, and mass profiles are renormalised by $E(z)^{-2}, K_{500}, P_{500}$, and $M_{500}^{Y_{X}}$, respectively.

clusters: A297, A963, and RXCJ1720.1+2638. These are all very well-known systems that appear in ROSAT-based X-ray catalogues, and they have all been observed in X-rays by $X M M$-Newton. They are detected at signal-to-noise ratios $S / N>$ 4.5 in the Planck survey (see Table 1).

We also examined data from the PSZ1 optical followup campaigns, in which a number of objects were identified as possible fossil systems (see Planck Collaboration Int. XXVI 2015; Planck Collaboration Int. XXXVI 2016). Applying the mass limit of $M_{500}^{Y_{z}}>3.75 \times 10^{14} M_{\odot}$ left us with two further candidates. During AO-14 we obtained XMM-Newton observations of one of them, PSZ1 G134.65-11.78, detected at a signalto-noise ratio of $S / N=4.8$ in the PSZ1. Only $25 \%$ of the observation is usable, and the X-ray emission is traced out only to $0.94 R_{500}$, but we include it here for completeness.

The X-ray observations, data reduction, and procedures to derive the various radial profiles are described in detail in Planck Collaboration Int. III (2013), where we investigated the scaling properties of these objects with their total SZ flux. Our $\mathrm{X}$-ray data set consists of density, temperature, pressure, entropy, and hydrostatic mass profiles of each object. These last were fitted with a Navarro-Frenk-White (NFW) profile to give an estimate of the concentration parameter $c_{500}$ and total mass $M_{500, \mathrm{HE}, \mathrm{NFW}}$; a second total mass estimate was obtained from iteration about the $M_{500}-Y_{\mathrm{X}}$ relation (Arnaud et al. 2010).

For the SZ data, we used thermal SZ maps obtained from the full 30-month Planck data set (Planck Collaboration XXII 2015) using the Modified Internal Linear Combination Algorithm (MILCA; Hurier et al. 2013). The SZ data reduction procedures are described in detail in Planck Collaboration Int. V (2013), where we investigated the stacked pressure profile of a sample of local clusters. Our SZ data set consists of a pressure profile for each object.

Table 1 lists various optical, X-ray, and SZ measurements for the four clusters, while Fig. 1 shows the radial profiles of density, entropy, pressure, and hydrostatic mass plotted on a logarithmic scale. Addition of the Planck data allows us to probe 
Table 1. Sample data.

\begin{tabular}{|c|c|c|c|c|c|c|c|c|c|}
\hline Cluster & $z$ & $\Delta m_{12}$ & $\begin{array}{r}T_{\mathrm{X}} \\
(\mathrm{keV})\end{array}$ & $\begin{array}{c}L_{\mathrm{X}} \\
\left(10^{44} \mathrm{erg} \mathrm{s}^{-1}\right)\end{array}$ & $\begin{array}{c}M_{500, \mathrm{HE}, \mathrm{NFW}} \\
\left(10^{14} M_{\odot}\right)\end{array}$ & $c_{500, \mathrm{NFW}}$ & $\begin{array}{c}M_{500}^{\mathrm{YX}_{\mathrm{X}}} \\
\left(10^{14} M_{\odot}\right)\end{array}$ & $f_{\mathrm{gas}, 500}$ & $S / N$ \\
\hline A267 & 0.227 & 2.12 & $5.48 \pm 0.15$ & $268+$ & $3.60_{-0.24}^{+0.26}$ & $2.81 \pm 0.28$ & $3.73_{-0.07}^{+0.07}$ & $0.12 \pm 0.02$ & 4.5 \\
\hline A963 & 0.206 & 2.20 & $9 \pm 0.12$ & ב & $\begin{array}{l}-0.34 \\
+0.52 \\
+0.52\end{array}$ & $3.12 \pm 0.45$ & $5.02_{-0.07}^{+0.07}$ & $0.12 \pm 0.02$ & 7. \\
\hline RXJ1720.1 & 0.164 & 1.90 & $5.80 \pm 0.12$ & $9.08 \pm 0.04$ & $5.26_{-0}^{+0.63}$ & $3.53 \pm 0.38$ & $5.30_{-0.08}^{+0.08}$ & $0.13 \pm 0.02$ & 10. \\
\hline PSZ1 G134.65-11.78 & 0.207 & 2.11 & $5.82 \pm 0.23$ & $4.99 \pm 0.05$ & $5.78_{-0.84}^{+0.98}$ & $3.09 \pm 0.48$ & $4.34_{-0.14}^{+0.08}$ & $0.10 \pm 0.02^{*}$ & 4.80 \\
\hline
\end{tabular}

Notes. Columns: (3): $r$-band magnitude gap between brightest- and second-brightest galaxies (Harrison et al. 2012; Zarattini et al. 2014, Burenin, priv. comm.); (4): X-ray temperature in the [0.15-0.75] $R_{500}$ region; (5) [0.1-2.4] keV X-ray luminosity; (6,7) mass and concentration from NFW fit; (8) mass from $M_{500}-Y_{\mathrm{X}}$ relation of Arnaud et al. (2010); (9) gas mass fraction (* extrapolated from $\left.0.94 R_{500}\right)$; (10) PSZ1 S/N.

significantly further out in the cluster volume than is possible with the X-ray data alone, reconstructing the radial pressure distribution out to $R \sim 2.5 R_{500}$ in each case.

\section{General radial profile characteristics}

The total masses are confirmed to be high, and lie in a narrow range at $M_{500}^{Y_{\mathrm{X}}} \sim 3.6-5.3 \times 10^{14} M_{\odot}$, as expected from the small spread in temperatures $(5-6 \mathrm{keV})$. For the three objects detected to at least $R_{500}$, the total masses derived from the NFW fit to the integrated mass profile agree with those estimated from the $M_{500}-Y_{\mathrm{X}}$ relation (Arnaud et al. 2010) to better than $4 \%$. This good agreement with a relation that was measured uniquely for relaxed systems would suggest that these fossil clusters may also be relaxed. The NFW concentration parameters also fall in a narrow range, and the mean concentration of $\left\langle c_{500}\right\rangle=3.2 \pm 0.4$ is the same as that measured for a sample of relaxed "normal" clusters by Pointecouteau et al. (2005). These fossil clusters are thus not over-concentrated relative to normal relaxed systems.

A general characteristic of the radial profiles shown in Fig. 1 is that three systems (A963, RXJ1720.1+2638, and PSZ1 G134.65-11.78) have remarkably similar profiles while those of A267 are clear outliers, especially within $0.2 R_{500}$. These three fossil systems have the highly peaked central density and declining central temperature profiles reminiscent of cool-core systems, while A267 has a flatter central density and temperature; indeed, its central density is an order of magnitude lower than that of RX J1720.1+2638. The entropy profiles converge towards the value expected from the non-radiative simulations of Voit et al. (2005) at $R_{500}$.

In the central regions, the pressure profiles have somewhat less dispersion than the density, and beyond $R>0.2 R_{500}$ they are remarkably similar; this is the case right out to the Planck detection limit of $R \sim 2.5 R_{500}$. The agreement between the X-ray and SZ pressure profiles is also good in the region of overlap. Beyond $R_{500}$, the pressure profiles of all objects except A267 marginally exceed the mean universal profile of Arnaud et al. (2010) but agree with that of Planck Collaboration Int. V (2013) at slightly more than $1 \sigma$.

\section{Gas mass fraction}

We took advantage of the extra radial reach afforded by the SZ observations to calculate the gas mass fraction by combining the SZ pressure and X-ray density profiles (e.g., Eckert et al. 2013). The gas pressure profile was obtained by fitting the X-ray and SZ data points (which together extend to $\sim 2.5 R_{500}$ ) with a generalised NFW model (Nagai et al. 2007), with the uncertainties estimated using a Monte Carlo procedure. The gas mass profile was obtained from the X-ray density profiles, which extend to $0.94-1.7 R_{500}$ depending on the object. The gas mass fraction profiles $f_{\text {gas }}(R)=M_{\text {gas }}(<R) / M_{\mathrm{HE}}(<R)$ were then calculated in

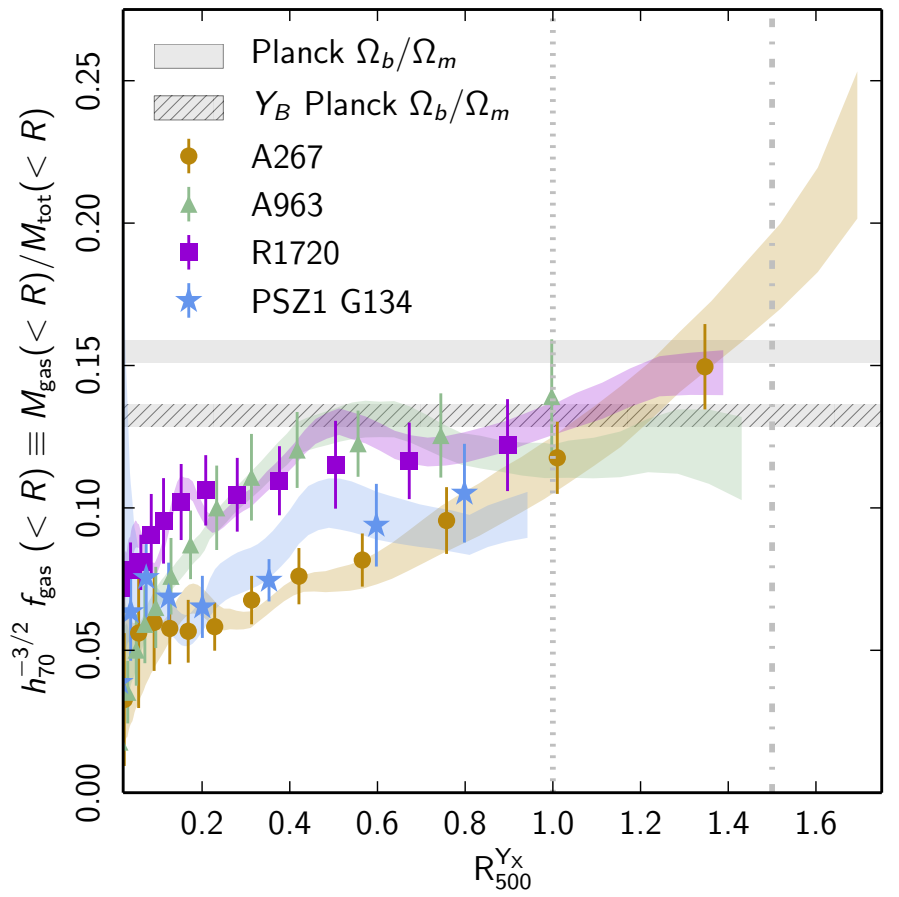

Fig. 2. Enclosed gas mass fraction profiles. Points with error bars indicate $f_{\text {gas }}$ obtained from the X-day data only; coloured envelopes show the $f_{\text {gas }}$ obtained from the X-ray-SZ pressure profile combined with the X-ray density profile (see Sect. 4). The solid grey line shows the cosmic baryon fraction measured by Planck Collaboration XVI (2014); $Y_{\mathrm{B}}=0.85$ is the baryon depletion factor at $R_{500}$ from the simulations of Planelles et al. (2013). The dot-dashed line indicates $R_{200}\left(\sim 1.5 R_{500}\right)$.

the radial range covered by the X-ray density profiles. In Fig. 2 the results obtained from combining the X-ray and SZ information are compared to those from the X-ray data only, plotted on a linear scale to accentuate the $R \gtrsim 0.2 R_{500}$ region. We also show the cosmic baryon fraction (Planck Collaboration XVI 2014), and the corresponding value corrected for the baryon depletion factor $Y_{B}=0.85$ from the simulations of Planelles et al. (2013).

These gas mass fraction profiles are striking. There is considerable diversity interior to $R_{500}$; within $R_{2500}$, RX J1720.1+2638 and A963 have 30\% more gas than A267; indeed, they appear to have attained the depletion-corrected cosmic baryon fraction by this radius, and flatten thereafter. The PSZ1 G134.65-11.78 profile also seems to flatten off at $R_{2500}$, but at a lower value of $f_{\text {gas }}$; it resembles those of RXJ1720.1+2638 and A963 but with lower normalisation. At $R_{500}$ the median value of $f_{\text {gas }, 500}=0.12 \pm 0.02$ agrees with recent observational (e.g., Lovisari et al. 2015; Ettori 2015) and theoretical (e.g., Le Brun et al. 2014) determinations, and with the depletioncorrected cosmic baryon fraction. 
Table 2. Dynamical indicators.

\begin{tabular}{lccc}
\hline \hline \multicolumn{1}{c}{ Cluster } & $\begin{array}{c}\text { Offset } \\
(\mathrm{kpc})\end{array}$ & $\langle w\rangle$ & $P_{\Delta}$ \\
\hline A267 & 33 & 0.008 & $<0.01$ \\
A963 & 12 & 0.003 & 0.86 \\
RX J1720.1+2638 & 2 & 0.004 & 0.52 \\
PSZ1 G134.65-11.78 & 12 & 0.006 & $\ldots$ \\
\hline
\end{tabular}

Notes. Columns: (2) offset between X-ray peak and BCG position; (3) standard deviation of the projected separations between the $\mathrm{X}$-ray peak and centroid in ten equally-spaced radii in [0.1-1] $R_{500}$; (4) Dressler-Shectman test probability. $P_{\Delta}<0.05$ is typical for disturbed systems (e.g., Pinkney et al. 1996).

Beyond $R_{500}$ the profiles diverge again; those of A963 and RXJ1720.1+2638 remain flat, while that of A267 continues to increase out to the detection limit, reaching $f_{\text {gas }}=0.21 \pm 0.01$ at $1.7 R_{500}$. This profile is highly reminiscent of that derived for Perseus by Simionescu et al. (2011). As pointed out by these authors, if there is significant radially dependent clumping of the gas density, then the X-ray data will overestimate the gas content because the quantity we are measuring is the average of the square of the electron density $\left\langle n_{\mathrm{e}}^{2}\right\rangle$, and not $\left\langle n_{\mathrm{e}}\right\rangle$. An $f_{\text {gas }} \sim 0.2$ would suggest an overestimate of the true gas density by a factor of 3-4, depending on how much the gas content is affected by depletion. An alternative explanation for the high apparent $f_{\text {gas }}$ is that it could be due to a departure from hydrostatic equilibrium $(\mathrm{HE})$ in the outer regions, with the hydrostatic mass underestimating the true mass owing to neglect of non-thermal pressure support. That A267's gas mass fraction profile continues to climb while those of A963 and RX J1720.1+2638 remain flat thus suggests a considerable variation in gas clumping and/or $\mathrm{HE}$ beyond $R_{500}$ in these fossil clusters.

\section{Discussion and conclusions}

Combining data from XMM-Newton and Planck, we have obtained the first high-quality measurements of the ICM of four bona fide fossil clusters out to unprecedented radii. Three systems have remarkably similar radial profiles reminiscent of coolcore systems. The fourth, A267, has the lowest inner gas content and shows the strongest evidence for clumping and/or departure from $\mathrm{HE}$ in the outskirts. It was classified as a fossil system by Eigenthaler \& Zeilinger (2009) and Zarattini et al. (2014).

We undertook a number of X-ray and optical measurements to ascertain the dynamical status of our objects, summarised in Table 2. With the X-ray data we measured the offset between the X-ray peak and BCG position, and the centroid shift parameter $\langle w\rangle$ (e.g., Poole et al. 2006; Pratt et al. 2009). In addition, we used the method proposed by Dressler \& Shectman (1988, the DS test) to probe for substructure in the galaxy distribution of A267, A963, and RX J1720.1+2638, for which extensive MMT/Hectospec and SDSS spectroscopic catalogues of $\sim 100-250$ members per cluster are available (Rines et al. 2013). A267 has the highest centroid shift value, although it would be classified as unperturbed but non-cool-core according to the REXCESS criteria of Pratt et al. (2009). Its X-ray image is slightly elliptical and the offset between the position of its X-ray peak and BCG, at $\sim 33 \mathrm{kpc}$, is more than three times that of the next most offset system. The DS test indicates that it is the only cluster that shows strong evidence of substructure in its galaxy distribution.

If fossils truly represent old, evolved systems, then one would expect higher than average NFW concentrations owing to their early formation time. The average $c_{500}$ of our four fossil systems is precisely the same as that obtained for "normal" local systems of a similar mass: they are not over-concentrated.

Assuming similar formation scenarios for low- and highmass fossils, our objects should have accreted the majority of their mass relatively early, and then remained undisturbed for a very long time. Growing today mainly through quiescent accretion, at fixed mass the hot gas in fossils would be in approximate $\mathrm{HE}$, their radial profiles would be identical, and there would be small system-to-system variations in clumping factors and/or departures from $\mathrm{HE}$ in the outer regions. The surprising variety in the radial gas content of the systems studied here appears to be driven by variations in gas content interior to $R_{500}$; conversely, outside $R_{500}$ the dispersion may be driven by variations in clumping and/or HE.

Overall, these observations suggest that fossil systems cannot all be the ultimate stage of gravitational collapse, and that while some high-mass fossil objects appear to be dynamically active even today (see also Zarattini et al. 2016), others are clearly relaxed but are not over-concentrated. Dedicated largevolume numerical simulations will be needed to better clarify the formation history of the fossil population, especially at the highest masses that we have probed here for the first time.

Acknowledgements. We thank R. Burenin, R. Barrena, J. Démoclés and J. A. Rubiño-Martín for their contributions to this project. This research has received funding from the European Research Council under the European Union's Seventh Framework Programme (FP7/2007-2013)/ERC grant agreement No. 340519. E.P. acknowledges the support of the French Agence Nationale de la Recherche under grant ANR-11-BS56-015.

\section{References}

Arnaud, M., Pratt, G. W., Piffaretti, R., et al. 2010, A\&A, 517, A92

Croston, J. H., Pratt, G. W., Böhringer, H., et al. 2008, A\&A, 487, 431

Dariush, A. A., Raychaudhury, S., Ponman, T. J., et al. 2010, MNRAS, 405, 1873

Dressler, A., \& Shectman, S. A. 1988, AJ, 95, 985

Eckert, D., Ettori, S., Molendi, S., Vazza, F., \& Paltani, S. 2013, A\&A, 551, A23 Eigenthaler, P., \& Zeilinger, W. W. 2009, Astron. Nachr., 330, 978 Ettori, S. 2015, MNRAS, 446, 2629

Harrison, C. D., Miller, C. J., Richards, J. W., et al. 2012, ApJ, 752, 12 Hurier, G., Macías-Pérez, J. F., \& Hildebrandt, S. 2013, A\&A, 558, A118 Jones, L. R., Ponman, T. J., Horton, A., et al. 2003, MNRAS, 343, 627 Khosroshahi, H. G., Maughan, B. J., Ponman, T. J., \& Jones, L. R. 2006, MNRAS, 369, 1211

Le Brun, A. M. C., McCarthy, I. G., Schaye, J., \& Ponman, T. J. 2014, MNRAS, 441, 1270

Lovisari, L., Reiprich, T. H., \& Schellenberger, G. 2015, A\&A, 573, A118 Nagai, D., Kravtsov, A. V., \& Vikhlinin, A. 2007, ApJ, 668, 1

Pinkney, J., Roettiger, K., Burns, J. O., \& Bird, C. M. 1996, ApJS, 104, 1 Planck Collaboration Int. III. 2013, A\&A, 550, A129

Planck Collaboration Int. V. 2013, A\&A, 550, A131

Planck Collaboration XVI. 2014, A\&A, 571, A16

Planck Collaboration XXIX. 2014, A\&A, 571, A29

Planck Collaboration Int. XXVI. 2015, A\&A, 582, A29

Planck Collaboration XXII. 2015, A\&A, submitted [arXiv: 1502.01596]

Planck Collaboration Int. XXXVI. 2016, A\&A, 586, A139

Planelles, S., Borgani, S., Dolag, K., et al. 2013, MNRAS, 431, 1487

Pointecouteau, E., Arnaud, M., \& Pratt, G. W. 2005, A\&A, 435, 1

Ponman, T. J., Allan, D. J., Jones, L. R., et al. 1994, Nature, 369, 462 Poole, G. B., Fardal, M. A., Babul, A., et al. 2006, MNRAS, 373, 881 Pratt, G. W., Croston, J. H., Arnaud, M., \& Böhringer, H. 2009, A\&A, 498, 361 Pratt, G. W., Arnaud, M., Piffaretti, R., et al. 2010, A\&A, 511, A85 Rines, K., Geller, M. J., Diaferio, A., \& Kurtz, M. J. 2013, ApJ, 767, 15 Simionescu, A., Allen, S. W., Mantz, A., et al. 2011, Science, 331, 1576 Sun, M., Forman, W., Vikhlinin, A., et al. 2004, ApJ, 612, 805 Voevodkin, A., Borozdin, K., Heitmann, K., et al. 2010, ApJ, 708, 1376 Voit, G. M., Kay, S. T., \& Bryan, G. L. 2005, MNRAS, 364, 909 Zarattini, S., Barrena, R., Girardi, M., et al. 2014, A\&A, 565, A116 Zarattini, S., Girardi, M., Aguerri, J. A. L., et al. 2016, A\&A, 586, A63 